\title{
Fractura mandibular múltiple en una zarigüeya (Didelphis marsupialis), estudio radiológico forense
}

\author{
Multiple mandibular fracture in a possum (Didelphis marsupialis), \\ forensic radiological study
}

Gallego-Rodriguez RS, Leysner-Tavera J, Aguirre JC. Fractura mandibular múltiple en una zarigüeya (Didelphis marsupialis), estudio radiológico forense. Rev Colombiana Cienc Anim. Recia. 2019; 11(1):Articulo682. DOI: https://doi.org/10.24188/recia.v11.n1.2019.682

Universidad de Sucre, Colombia

Los autores permiten a RECIA reimprimir el material publicado en él. En caso de que un autor quiera traducir o usar una publicación parcial o completa de nuestro Diario, el autor debe obtener un permiso por escrito del editor de la revista.

Revista Colombiana de Ciencia Animal - RECIA está distribuido bajo una Licencia Creative Commons Atribución-CompartirIgual 4.0 Internacional. 


\title{
Fractura mandibular múltiple en una zarigüeya (Didelphis marsupialis), estudio radiológico forense
}

\author{
Multiple mandibular fracture in a possum (Didelphis marsupialis), forensic radiological \\ study
}

Renso Sneider Gallego-Rodriguez

DOI: https://doi.org/10.24188/recia.v11.n1.2019.682

Corporación Universitaria Remington, Facultad de Medicina

Veterinaria, Grupo de Investigación en Veterinaria (GINVER),

Medellín, Antioquia, Colombia.

(iD https://orcid.org/0000-0003-1563-9731

renso.gallego@uniremington.edu.co

\section{Jesika Leysner-Tavera}

Corporación Universitaria Remington, Facultad de Medicina Veterinaria, Grupo de Investigación en Veterinaria (GINVER), Medellín, Antioquia, Colombia.

(D) https://orcid.org/0000-0003-2729-4195

jesika.leysner@gmail.com

Julio Cesar Aguirre

Corporación Universitaria Remington, Facultad de Medicina

Veterinaria, Grupo de Investigación en Veterinaria (GINVER),

Medellín, Antioquia, Colombia.

iD https://orcid.org/0000-0002-0998-3328

veterinarioforense@gmail.com

Recepción: 17 Noviembre 2018

Aprobación: 31 Mayo 2019

Publicación: 13 Junio 2019

\section{RESUMEN}

El atropellamiento de las especies silvestres es una de las principales causales de la mortalidad para estos animales, disminuyendo drásticamente su población. Se recibe una zarigüeya (Didelphis marsupialis) en la clínica veterinaria de la Universidad Remington de Medellín - Antioquia, con historial de politraumatismo reciente por atropellamiento con signos evidentes de dolor, múltiples lesiones lacerantes y abrasivas en la región craneal, torácica y coxal, el paciente inicia manejo médico farmacológico, fluidoterapia y es hospitalizado; debido a la gravedad de las lesiones el animal muere y se decide realizar un estudio radiológico ortogonal (vistas laterales y ventro dorsales de cráneo, tórax y abdomen, laterales de esqueleto apendicular) en el cual se observa fractura en tallo verde de la rama horizontal mandibular izquierda, en el ángulo mandibular izquierdo se evidencia fractura oblicua completa, en la porción del hueso maxilar izquierdo se observa una fractura longitudinal levemente desplazada.

Palabras clave: Atropellamiento, fauna silvestre, radiología veterinaria, zarigüeya 


\begin{abstract}
The death by vehicular collision is one of the main causes of death in wildlife, drastically affecting the population number of several species. A Zarigueya (didelphis marsupialis) is received in the veterinary clinic of the Remington University in Medellín - Antioquia, with a recent history of multiple trauma with obvious signs of pain, multiple laceration and abrasive lesions in the cranial, thoracic and coxal regions. The patient starts medical pharmacological management, fluid therapy and is hospitalized; past few hours the patient died due to the severity of the lesions. It is decided to perform an orthogonal radiological study (lateral and ventro - lateral views of the skull, thorax and abdomen, laterals of the appendicular skeleton) in which a fracture is observed in the green stem of the horizontal left mandibular branch, in the left mandibular angle a complete oblique fracture is observed and in the left maxillary bone there is a slightly displaced longitudinal fracture.
\end{abstract}

Keywords: Run over, wildlife, veterinary radiology, opossum, road ecology, forensic.

\title{
INTRODUCCIÓN
}

La interfaz urbano forestal es un factor común en la presentación de eventos traumáticos o muertes por atropellamiento para la fauna silvestre (1), por este motivo se hace necesario el estudio imagenológico en el diagnóstico postmortem, ya que permite evidenciar lesiones que relacionadas con la muerte del paciente (2).

La obtención de un estudio radiológico postmortem antes de la necropsia debe considerarse un protocolo estándar en la medicina veterinaria forense ya que permitirá el reconocimiento de hallazgos sin realizar un abordaje invasivo (3). Las indicaciones en el uso de la radiografía en pacientes postmortem se indican en determinación del género, identificación de la edad y en eventos relacionados con la mueres, a su vez, se relacionan con casos de maltrato físico, de heridas cortopunzantes que puedan generar presencia de gas a la imagen, con lesiones por proyectiles balísticos y con politraumatismos por atropellamiento (4).

Las carreteras y el tráfico vehicular es un factor relevante para la mortalidad de fauna silvestre ya que afecta rutas naturales migratorias de mamíferos terrestres y fragmenta el área de actividad y territorios. La mortalidad directa se debe a colisiones lo cual reduce las poblaciones en las áreas de menor densidad, de manera secundaria generara cambios en el ecosistema y deterioro en las poblaciones naturales (5).

En un estudio hecho por (6) reportó que 356 animales murieron por atropellamiento en la autopista del pacífico en Australia, a su vez, Fonseca., (7) menciona que 83 animales mamíferos murieron por la misma causa en la autopista 441 en Florida, Estados Unidos, de estas especies se describe que una de las más afectadas fue la Zarigüeya (Didelphis marsupialis) (7).

En un estudio realizado en el Departamento de Sucre, Colombia encontraron que de un total de 431 animales muertos por atropellamiento, el 28.1\% (121 animales) eran mamíferos, a su vez, el 6.1\% (26 animales) pertenecían a la especie Didelphis marsupialis (8). A nivel de departamentos como Antioquia se relaciona con una alta mortalidad debido a colisiones; en países como Brasil y Venezuela es la especie más afectada por atropellamiento (9).

\section{Descripción del Caso}

Se recibe en la clínica veterinaria de la universidad Remington, una chucha común (Didelphis marsupialis), de sexo hembra, adulta, con $2.1 \mathrm{Kg}$ de peso, con historial reciente de politraumatismo debido a atropellamiento vehicular. La evaluación clínica inicia con el examen físico y dentro del plan diagnóstico se realizó un estudio radiológico. A la auscultación cardiaca no se puede determinar la frecuencia debido a la taquicardia severa que presentaba el animal, la frecuencia respiratoria fue de $50 \mathrm{rpm}$, el paciente presenta actitud deprimida y letárgica, anorexia, múltiples heridas lacerantes y 
abrasivas en el cráneo sobre el aspecto rostral y temporal, a nivel de ambos hemitórax y en la región coxal, debido a la complejidad de sus lesiones el paciente fallece durante los procedimientos médicos clínicos (cateterización, establecimiento de fluidoterapia y reanimación cardiovascular).

Como parte del diagnóstico imagenológico forense se decide realiza un estudio radiológico (equipo generador VR 40 VET_RAY INC, USA, Idaho 1989) basado en un estudio ortogonal del paciente, en el que se utilizó un kilovoltaje de 60 con un amperaje de 16 y un miliamperaje de 0.16; se tomaron: vista lateral completa (ver figura 1), vista lateral de tórax - abdomen y de cráneo, vista ventro dorsal de tórax - abdomen, y cráneo, a su vez, se realizan radiografías laterales y antero - posteriores de los miembros, como hallazgo anexo se observa un puente intervertebral (espondilo artrosis) entre los cuerpos vertebrales de la 1 y la 2 vertebral caudal.

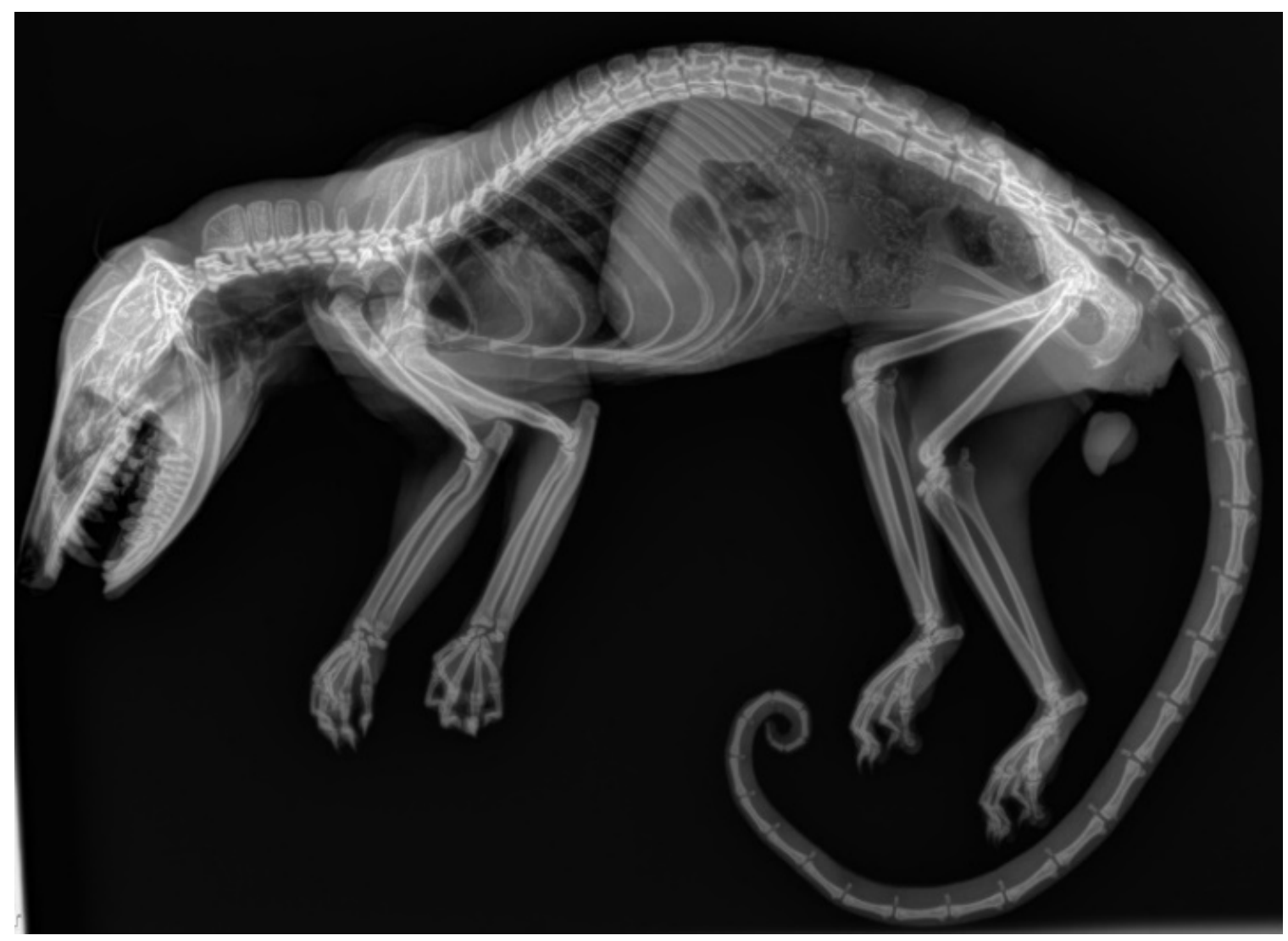

Figura 1. Estudio radiológico lateral completo post-mortem de paciente Didelphis marsupialis en el cual no se observa ninguna lesión a nivel óseo ni de tejido blando, por medio de esta radiografía se descartan fracturas del esqueleto axial y apendicular.

En el estudio radiológico ventro dorsal de cráneo se observa fractura en tallo verde de la rama horizontal mandibular izquierda, en el ángulo mandibular izquierdo, se evidencia fractura oblicua completa, en la porción del hueso maxilar izquierdo se observa una fractura longitudinal levemente desplazada (Figura 2). 


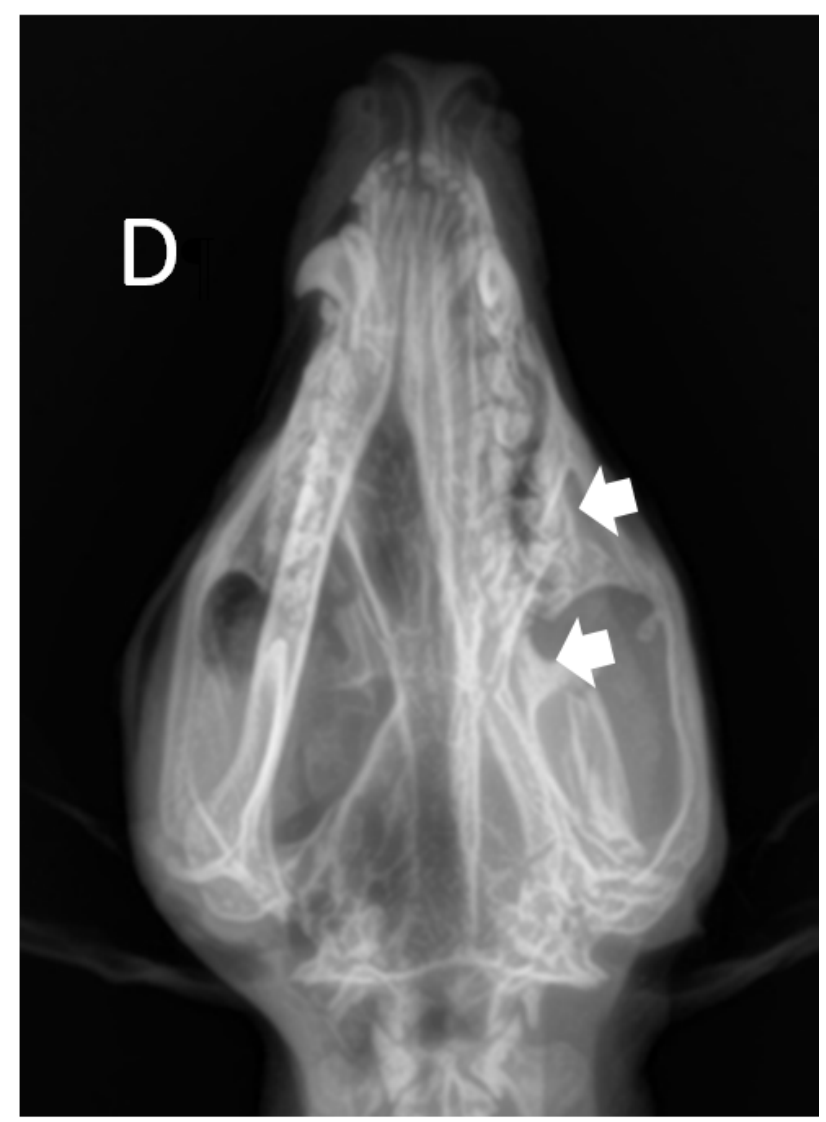

Figura 2. Estudio radiográfico ventro dorsal de cráneo en el cual se observan múltiples fracturas en el aspecto izquierdo de la región maxilar y mandibular (flechas).

Las lesiones sobre este individuo se encontraron reunidas en su mayoría sobre el eje estomatognático lo cual, en conjunto con la naturaleza de las mismas, es indicativo de un evento de alta transferencia de energía en poco tiempo (segundos) debido a las fuerzas que actuaron sobre el cuerpo del animal en el momento (10).

\section{DISCUSIÓN}

En la medicina veterinaria la radiología forense es un campo que ha ido creciendo debido a los aportes realizados por médicos veterinarios, peritos forenses veterinarios, patólogos y en la actualidad imagenólogos los cuales por la correlación de los hallazgos evidencian las lesiones y las causas probables de muerte de un paciente (11). La fauna silvestre es una de las poblaciones animales más vulneradas siendo los accidentes por atropellamiento una de las causas de muerte más común (12)Sucre a Mar】 u00eda la Baja, Bol \u00edvar, en \u00e9poca seca y \u00e9poca de lluvias; con longitud de $49 \mathrm{Km}$, la $\mathrm{v} \backslash \mathrm{u} 00 \mathrm{eda}$ bordea la porci $\backslash \mathrm{u} 00 \mathrm{f} 3 \mathrm{n}$ noroccidental de los Montes de Mar $\backslash \mathrm{u} 00 \mathrm{eda}$, amplia zona de bosque seco tropical remanente del Caribe colombiano. Se trabaj $\backslash$ u00f3 durante 6 meses continuos de octubre de 2014 a marzo de 2015, con dos recorridos/ semana para un total de 48 muestreos, realizados de las 05:00 a las 08:00 horas, con velocidad media de $15 \mathrm{~km} /$ hora. Las muestras fueron identifi cadas in situ. Al comparar estad $\backslash \mathrm{u} 00$ edsticamente las dos $\backslash \mathrm{u} 00 \mathrm{e} 9$ pocas muestreadas respecto de los atropellamientos totales se determina que no existe diferencia signifi cativa (DS=13,213, $t=0,468, d f=31, p=0,642$. Por tal motivo, la utilización de esta técnica permite al médico veterinario tener herramientas sobre el diagnóstico y posible causa de muerte en un paciente (13).

Según Kozinc Z en 2017 (10) menciona que en el atropellamiento se evidencian manifestaciones físicas las cuales provocan evidenciadas sobre la corporalidad del individuo evaluado. Los hallazgos se describen como politraumatismo contundente en cercana relación al deceso sin presencia de lesión patrón por lo cual se podría inferir el uso o participación de elemento de morfología irregular de alta carga cinética y guarda vinculación causal con atropellamiento con vehículo automotor. 
En los hallazgos radiológicos postmortem encontrados en este individuo, se evidencia que las lesiones más relevantes se encuentran en el tejido óseo afectándose principalmente la región craneal del animal, en la cual se observan múltiples fracturas relacionadas con el aspecto mandibular izquierdo, contrario a lo mencionado por Watson en el 2017, el cual describe que es más común que el reporte de hallazgos radiológicos en el esqueleto apendicular de fauna silvestre muerta por atropellamiento (3).

Según un informe sobre accidentes con vehículos automotores realizado por el Instituto Colombiano de Medicina Legal y Ciencias Forenses Contreras en el 81\% de los casos analizados la interacción ocurrió entre la parte delantera del vehículo con el otro objeto (14). Este resultado revela la naturaleza frontal de los accidentes de vehículos en los casos analizados y está en consonancia con lo reportado por Contreras et al para la línea de visión preimpacto del conductor respecto al otro objeto (14). Las colisiones con las partes laterales de los vehículos se presentaron en el $9 \%$ de los casos analizados, que supera los $2.7 \%$ encontrados en los estudios de Bosio et al (15). Tan sólo un 2\% de los casos analizados correspondió a impacto en la parte trasera del vehiculo, resultado que está en consonancia con el 3\% de los estudios de Bosio et al (15). La lesión entonces dependerá de la velocidad y la cantidad de energía transmitida, el área de superficie sobre la cual la energía es aplicada, y las propiedades elásticas de los tejidos sobre los cuales se transfiere la energía aplicada (15).

Las carreteras han sido un problema recurrente para los animales cuyo habitad ha sido arrebato o transformado (16). Entre los efectos ecológicos más significativos podemos destacar la fragmentación de los ecosistemas, dispersión de especies exóticas y disminución de las poblaciones de especies de fauna y flora, producción de ruido y contaminación de las aguas y suelo (1). El atropellamiento de fauna es uno de los efectos más relevantes para el ecosistema ya que es común encontrar en las carreteras animales atropellados poli traumatizados que se desplazan y mueren en otro lugar sin poder registrar la muerte del animal (8).

A nivel mundial se han llevado a cabo distintas investigaciones alrededor del tema, países como Estados Unidos y países Europeos y Australia hacen referencia sobre la cantidad de atropellamientos presentados en sus vías y la evidente amenaza que esto provoca en las especies que allí habitan (1) . En los Estados Unidos, el Centro de Investigación para la Vida Silvestre ha estimado que diariamente es atropellado un millón de animales en todas las autopistas del país (12)Sucre a Mar \u00eda la Baja, Bol \u00edvar, en \u00e9poca seca y \u00e9poca de lluvias; con longitud de $49 \mathrm{Km}$, la v\u00eda bordea la porci \u00f3n noroccidental de los Montes de Mar\u00eda, amplia zona de bosque seco tropical remanente del Caribe colombiano. Se trabaj $\backslash$ u00f3 durante 6 meses continuos de octubre de 2014 a marzo de 2015, con dos recorridos/ semana para un total de 48 muestreos, realizados de las 05:00 a las 08:00 horas, con velocidad media de $15 \mathrm{~km} /$ hora. Las muestras fueron identifi cadas in situ. Al comparar estad $\backslash \mathrm{u} 00$ edsticamente las dos $\backslash \mathrm{u} 00 \mathrm{e} 9$ pocas muestreadas respecto de los atropellamientos totales se determina que no existe diferencia signifi cativa $(D S=13,213, t=0,468$, $\mathrm{df}=31, \mathrm{p}=0,642$.

En un estudio realizado entre 2001 y 2002 (17) en un estudio realizado se reportó 83 mamíferos atropellados entre los cuales se encontraron Ratas, zarigüeyas, coyotes, armadillos, venados, mapaches, zorros, nutrias, conejos, entre otros (17). Allí se determinó que el número de mamíferos atropellados es menor respecto al número de aves además de los anfibios y reptiles.

Se realizó un estudio en el Municipio de Envigado-Antioquia durante los años de 2008 y 2013 (5) en el cual se describió la fauna silvestre afectada por atropellamiento en la vía del Escobero del municipio de Envigado - Antioquia, se reportaron un total de 35 animales muertos por atropellamiento en la carretera, de los animales afectados la especie Zarigueya (Didelphis marsupialis) presentó uno de los mayores valores con un total de 17 animales, se considera que la tasa de fauna silvestre afectada disminuyó ya que anteriormente entre los años 2000 y 2006 se hizo un estudio donde se reportan 44 animales (5). Los mamíferos son predisponentes a este tipo de eventos, donde la tasa aumenta más en época de apareamiento ya que deben cruzar la vía para encontrarse con hembras o machos de su especie, los animales jóvenes son inexpertos en la búsqueda de alimento y son susceptibles a ser atropellados (5).

Las medidas de compensación instauradas por los diferentes departamentos de Ambiente no han logrado minimizan el impacto ecológico que la construcción de carreteras trae a los animales 
principalmente porque no se realizan estudios previos a la construcción evaluando los posibles daños que pueden traer al ecosistema si no después es cuando se hace evidente el impacto que esto trae a los diferentes animales provocando en algunos casos la perdida de especies nativas (1).

\section{REFERENCIAS}

1. Arroyave M, Gómez C, Gutiérrez ME, Múnera DP, Zapata PA, Vergara IC, et al. Impactos de las Carreteras sobre la Fauna silvestre y sus principales medidas de manejo. Rev EIA. 2006; 3(5):4557. https://revistas.eia.edu.co/index.php/reveia/article/view/146

2. Bosio LA, Cohen RV, Ramos NL. Accidentología vial: elementos de estudio forense. Vol. 1. Cuadernos de Medicina Forense: Argentina; 2009. https://www.csjn.gov.ar/cmfcs/files/pdf/ CMFA-Tomo1(2009)/CMFA1-1-Bosio.pdf

3. Cooper JE, Cooper ME. Introduction to veterinary and comparative forensic medicina. Arnes: Blackwell Publishinf Profesional 2007. https://onlinelibrary.wiley.com/doi/ book/10.1002/9780470752944

4. De La Ossa J, Galván-Guevara S. Registro de mortalidad de fauna silvestre por colisión vehicular en la carretera Toluviejo - ciénaga La Caimanera, Sucre, Colombia. Biota Colomb. 2015; 16(1):6876. http://revistas.humboldt.org.co/index.php/biota/article/view/368

5. Delgado-VC. Additions to mammals killed by motor vehicles in vía of El Escobero, Envigado (Antioquia). Rev EIA. 2014; 11(22 2):147-153. URL Available in: https://revistas.eia.edu.co/ index.php/Reveiaenglish/article/view/949

6. Elifritz JM, Nolte KB, Hatch GM, Adolphi NL, Gerrard C. Forensic Radiology. Pathobiol Hum Dis A Dyn Encycl Dis Mech. 2014; 3448-58. https://doi.org/10.1016/B978-0-12-386456-7.06706-X

7. Fonseca ACB, Massad MRR, Massad L, Outi Baroni C, Missen T, Reis STJ, et al. Post-mortem computed tomography angiography and forensic necropsy of a brown howler monkey: A case report. J Forensic Radiol Imaging. 2017; 8:48-51. https://doi.org/10.1016/j.jofri.2017.03.006

8. Kozinc Ž, Šarabon N. Common Running Overuse Injuries and Prevention. Monten. J. Sports Sci. Med. 2017; 6(2). https://doi.org/10.26773/mjssm.2017.09.009

9. Lora L, Barichivich J, Staiger S, Smith KC. Detection Probabilities and Site Occupancy Estimates for Amphibians at Okefenokee National Wildlife Refuge. Am Midl Nat. 2018; 155(1):149-61. https://doi.org/10.1674/0003-0031(2006)155[0149:DPASOE]2.0.C0;2

10. Monroy MC, De La Ossa-lacayo A, De La Ossa VJ. Tasa De Atropellamiento De Fauna Silvestre En La Vía San Onofre - María La Baja, Caribe Colombiano. Rev Asoc Col Cienc. 2015; 1(27):8895. http://www.asociacioncolombianadecienciasbiologicas.org/ojs/index.php/accb/article/ view/106/106

11. Munro R, Munro H. Forensic veterinary medicine 2. Postmortem investigation. In Pract. 2011; 33(6):262-70. http://dx.doi.org/10.1136/inp.d3599

12. RodríguezCG.Accidentes demotociclistas.Hacialaidentificacióndemedidasefectivas. Institutode Medicina Legal: Colombia; 2010. http://www.medicinalegal.gov.co/documents/20143/49508/ Accidentes+De+Transito.pdf

13. Singal K. History and Modalities of Forensic Radiology: A Review. Int J Adv Multidiscip Res. 2015; 2(12):11-13. DOI: https://dx.doi.org/10.21088/ijfmp.0974.3383.8415.5 
14. Taylor BD, Gold Ingay R. Wildife roadkills on three major roads in North-Eastern New South wales. Wildl Res. 2004; 31:83-91. https://doi.org/10.1071/WR01110

15. Watson E, Heng HG. Forensic Radiology and Imaging for Veterinary Radiologists. Vet Radiol Ultrasound. 2017; 58(3):245-58. https://doi.org/10.1111/vru.12484

16. Bauni JAFS. Mortalidad de fauna silvestre por atropellamientos en el bosque atlántico del Alto Paraná, Argentina. Ecosistemas. 2017; 26(3):54-66. https://doi.org/10.7818/ECOS.2017.26$\underline{3.08}$

17. Lora L, Barichivich J, Staiger S, Smith KC. Detection Probabilities and Site Occupancy Estimates for Amphibians at Okefenokee National Wildlife Refuge. Am Midl Nat. 2018; 155(1):149-161. https://pubs.er.usgs.gov/publication/70031177 